\title{
Isochrones, Traffic and DEMOgraphics
}

\author{
Alexandros Efentakis \\ Research Center "Athena" \\ Artemidos 6, Marousi 15125, Greece \\ efentakis@imis.athena-innovation.gr
}

\author{
Nikos Grivas \\ Research Center "Athena" \\ Artemidos 6, Marousi 15125, Greece \\ grivas@imis.athena-innovation.gr
}

\section{George Lamprianidis \\ Research Center "Athena"}

Artemidos 6, Marousi 15125, Greece

glampr@imis.athena-innovation.gr

\author{
Georg Magenschab \\ WIGeoGIS GmbH \\ Hansalgasse 3, 1030 Vienna, Austria \\ gm@wigeogis.com
}

\author{
Dieter Pfoser \\ Department of Geography and \\ GeoInformation Science \\ George Mason University \\ 4400 University Drive, MS 6C3 \\ Fairfax VA 22030-4444 \\ dpfoser@gmu.edu
}

\begin{abstract}
Catchment area and reachability analysis, i.e., the area from which a location attracts visitors and the minimum distance to a target location, respectively, are interesting problems when studied in the context of time-parameterized networks, such as road networks affected by traffic. This work utilizes live-traffic assessment results produced by Floating Car Data and their application to such crucial geomarketing test cases. We combine state-of-the-art isochrone computation utilizing live-traffic and demographics data to provide efficient catchment area and reachability calculations. The online demo presented here, showcases the critical impact of live-traffic assessment on business intelligence decisions related to space.
\end{abstract}

\section{Categories and Subject Descriptors}

H.2.8 [Database Management]: Database Applications—Spatial databases and GIS; H.3.5 [Information Storage and Retrieval]: Online Information Services-Web-based services

\section{General Terms}

Design

\section{Keywords}

Geormaketing, Isochrones, Traffic, Demographics, FCD

\section{INTRODUCTION}

Our latest research efforts of [6] aim towards applying state-ofthe-art research about Floating Car Data, map-matching, historic speed profile computation, live-traffic assessment and time dependent shortest path computation to provide cost-effective fleet management solutions. This process required several intermediate steps

\footnotetext{
*On leave from Research Center "Athena”, Greece.
}

Permission to make digital or hard copies of part or all of this work for personal or classroom use is granted without fee provided that copies are not made or distributed for profit or commercial advantage and that copies bear this notice and the full citation on the first page. Copyrights for thirdparty components of this work must be honored. For all other uses, contact the Owner/Author.

Copyright is held by the owner/author(s).

SIGSPATIAL'13, Nov 05-08 2013, Orlando, FL, USA

ACM 978-1-4503-2521-9/13/11.

http://dx.doi.org/10.1145/2525314.2525325 such as: Creating road network graphs from OpenStreetMaps data, collecting a large amount of Floating Car Data (FCD) from fleet vehicles, applying state-of-the-art map matching algorithms on this data and consequently producing high-quality historic speed profiles along with frequently updated live-traffic assessment. This combination of live-traffic information and speed profiles will be subsequently used to provide up-to-date live-traffic shortest path computation (updated every 5 minutes).

As a result of these efforts, our integrated SimpleFleet service is fully operational for three European cities namely: Athens (Greece), Berlin (Germany) and Vienna (Austria). Floating Car Data (FCD) for Vienna and Berlin originate from taxi fleets of 2000-5000 vehicles respectively, where for Athens FCD is provided from two commercial fleets of 1000 vehicles each. The online demo of [2] and [3] clearly demonstrated the huge potential of our effort.

Although our main focus in [6] is mainly towards fleet management, the live-traffic assessment results produced, may prove extremely beneficial to other contexts as well. Such a context is geomarketing, i.e., the integration of geographical information into various aspects of business intelligence, such as marketing, sales and distribution. A crucial component in the effort of integrating traffic information in a geomarketing context is the concept of isochrones, which are informally defined as the area from which a specific point of interest is reachable within a given time interval. In this sense, isochrones may provide accurate information about where to build a new franchise store in order to reach a larger pool of customers or identify areas less covered by existing stores. Since we are able to provide state-of-the-art isochrone computation based on live-traffic, this increased accuracy offers a unique advantage in comparison to typical static road network approaches.

In this spirit, we developed an additional geomarketing demo (referred hereafter as SimpleFleet geomarketing demo) that combines live-traffic, isochrones and demographic / business data for Berlin and Vienna in order to demonstrate the actual impact of traffic fluctuations to business intelligence decisions. The geomarketing demo presented here, supports most modern Web-browsers and is available at http://webgistu.wigeogis.com/protozone/gm/ simplefleet/start.php.

The outline of this work is as follows: Section 2 describes our scientific contribution beyond the current state-of-the-art. The implementation details of the demo are provided in Section 3. The actual demo and its interface are presented in Section 4 along with a summary of of the results obtained by the application and their 
significance. Finally, Section 5 gives conclusions and provides directions for future work.

\section{OUR CONTRIBUTION}

Isochrones are defined in [1] as the "set of all points from which a specific point of interest is reachable within a given time span". An important paper for the concept of isochrones and related to our work is [5], since it was the first to claim that the whole spatial area covered by an isochrone is important. In addition, that work introduced the "Edges' Hull" algorithm which creates a single area which is defined by a polygon composed of the outermost edges of the isochrone network. This approach offers increased accuracy in comparison to previous, typical convex hull approaches.

Although isochrones have been utilized in public transport and walking combinations in [1] and [4], to the best of our knowledge we were the first to combine the state-of-the-art isochrone computation of [5] with real live-traffic data (refreshed every five minutes). This functionality was already documented in [2] and [3]. Our aforementioned SimpleFleet service also features a Visualization API exposing its core functionality, so that isochrone computation may also be used by third-party apps.

In this work, we utilize the Visualization API and further expanded previous results by combining the live-traffic isochrones with demographics / business data for Berlin and Vienna provided by business partner WIGeoGIS. Hence, we are the first to provide accurate, substantial but most of all quantitive evidence (based on our live-traffic data, instead on relying on third party sources) about the impact of traffic in business intelligence decisions. Our results clearly show that this impact is much bigger than what it was expected (see Sec. 4.2). Therefore, approaches that either: a) ignore traffic by assigning typical static speeds per road class or b) rely on conventional convex hull approaches, are inherently imprecise by a big margin, since they greatly overestimate the area from which a specific point of interest is reachable.

Methodology. The SimpleFleet service provides access to its data via a simple REST endpoint (i.e., the Visualization API), which is publicly accessible. For the purposes of the geomarketing demo, live-traffic isochrone data for Vienna and Berlin was requested in regular intervals of 15 minutes, from April 26th 2013 until May 31 st 2013, using the provided API. Each such isochrone request returns six isochrones with travel times starting from 5 minutes (for the first isochrone) and a 5 minutes step, so that the last isochrone covers a travel time span of 30 minutes.

All six isochrone multi-polygons are subsequently stored in a database along with a timestamp and a location identifier (Berlin, Vienna). Then a separate offline process takes place and calculates the values for population, households, banks and retail stores for each isochrone record. The calculated value is merely a count, derived by overlaying each isochrone multi-polygon with a $250 \times 250 \mathrm{~m}$ raster containing population data. Banking and retail store information is extracted from business directories for each city and were provided by partner WIGeoGIS.

Since a picture is worth a thousand words the better way to showcase these results is through an intuitive Web application publicly available. With that purpose in mind, in the next section we will describe the several geospatial and Web technologies required for actually building and servicing our geomarkerting demo.

\section{SYSTEM IMPLEMENTATION}

Our demo web application is built with standard modern technologies for managing and displaying spatiotemporal data online. For our persistent storing mechanism, we utilized a Postgis enabled
PostgreSQL database. PostgreSQL is a well known, powerful open source relational database management system. In addition, PostGIS "spatially enables" the PostgreSQL server, allowing it to be used as a backend spatial database for geographic information systems. Hence, their combination provides excellent performance and efficient spatial calculations.

The Javascript front-end of the demo is designed with DHTMLX, a rich Javascript library that delivers a complete set of UI components and has built-in AJAX support for fetching data asynchronously from the remote server. The bar chart (Fig.1) displaying the data, uses D3JS, which is a powerful Javascript library for manipulating documents based on data. The interactive map is powered by OpenLayers, a free Javascript mapping framework that allows creation of dynamic maps on any web page. The map theme is called "Toner" and was designed by "Stamen" (a design and technology studio based in San Francisco). Still, the OpenLayers library would easily work with any other available WMS source.

The demo's front-end consumes data from the database via simple PHP scripts, using AJAX requests. To fetch the corresponding data, each request requires the following four parameters:

- The travel time span of the isochrones in minutes $(5,10$, $15, \ldots, 30 \mathrm{~min})$

- A specific date (between April 26th, 2013 until May 31st, 2013 - the time period covered by the demo)

- A time range within the specified date (e.g., between 08:00 and 17:30)

- The demographic / business data type requested (population, households, banks or retail stores)

As described in Section 2 all available data is stored in a single table in the underlying PostgreSQL database, so the query may efficiently select all records matching the user's specified criteria.

The corresponding server's response is an array of objects (JSON format), each containing the following information:

- The timestamp value,

- The demographic / business data absolute count,

- The demographic / business data average count,

- The delta between the average and the absolute count,

- The travel time of the isochrone $(5,10,15, \ldots, 30 \mathrm{~min})$

- The ID of the stored isochrone multi-polygon in the database.

The isochrone itself is not included in the response for bandwidth and performance reasons. To actually draw the isochrone on the map, when required, another AJAX call is made, using the isochrone ID as its only parameter. The multi-polygon returned is encoded using the WTK (Well Known Text) representation.

\section{ONLINE APPLICATION}

The geomarketing demo is an interactive web application that showcases one of the many potential uses of the produced results of the SimpleFleet service, in scenarios not directly related to fleet management. Population, households, banks and retail stores data is correlated with isochrones to provide valuable information, such as catchment areas (for population and household data) and reachability analysis (for bank branches and retail stores). The demo focuses on this kind of data and intuitively demonstrates how it changes over time through the fluctuation of traffic; within a month period or even during a certain day. This visual representation easily showcases the benefits of computing isochrones using livetraffic data as opposed to a typical traffic-less static road network approaches. 


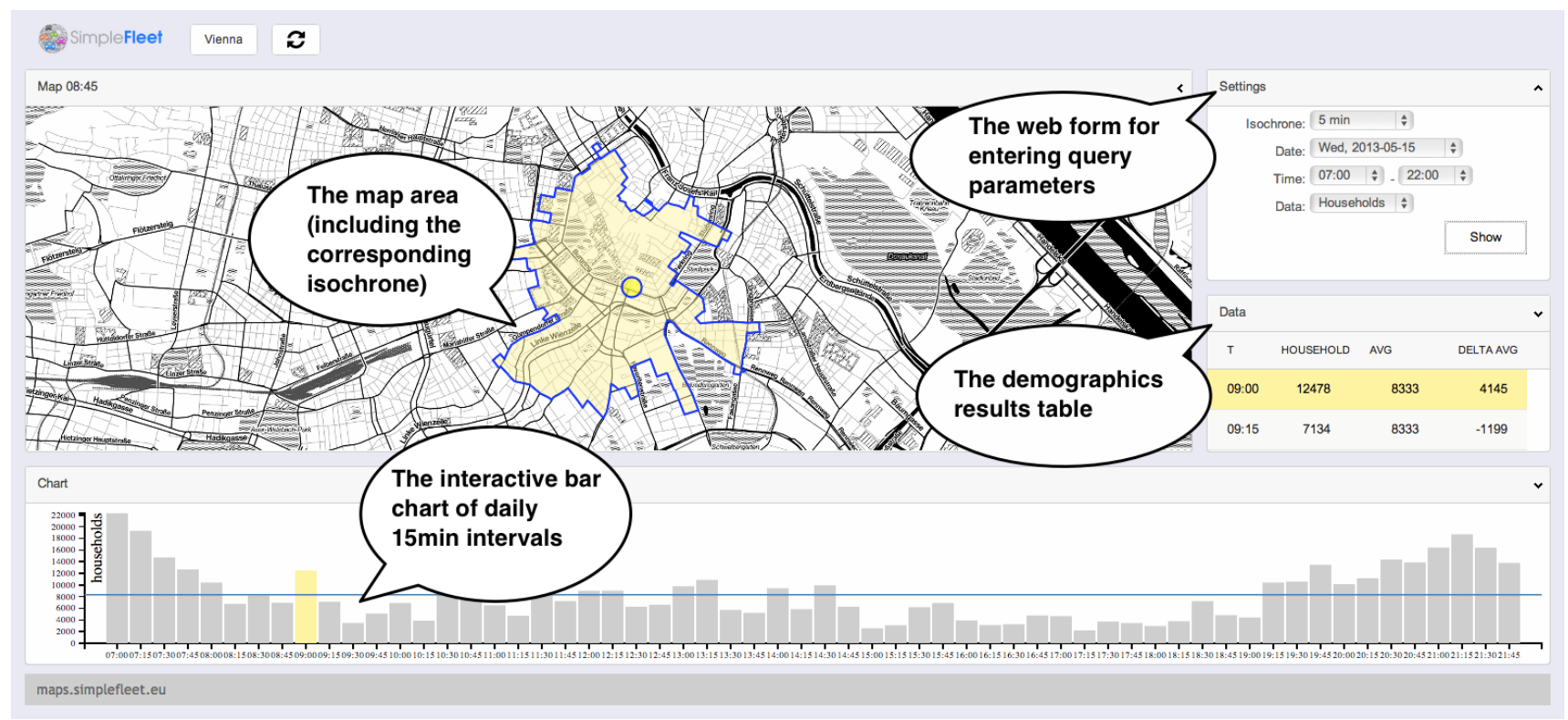

Figure 1: Vienna city center, on Wednesday 15th May 2013, at 09:00 am. Approximately 12k households are reachable within five minutes from the city center at that time.

\subsection{Interface}

The demo is available online as a single page web-application. When it loads up, the demo's interface is divided into four main areas (see Figure 1):

- A web-form for setting the query / isochrones parameters

- An interactive bar chart of daily $15 \mathrm{~min}$ intervals

- The demographics results table, displaying the demographic/ business data contained inside the isochrone area

- The map area (including the isochrone multi-polygon)

On the top of the page there is a drop-down menu that allows the user to choose between the two available cities: Vienna (set as the default area) and Berlin. The user may set the desired options / request parameters (see Section 3), in the web form located in the upper right corner of the screen and click the "Show" button. This action will submit the form via AJAX and return the respective de- mographic / business counts requested. When the AJAX call completes, the newly retrieved data is displayed in the table panel and simultaneously gets graphed in the interactive bar chart area located at the bottom. In the bar chart area, the horizontal axis represents the timestamp during the selected day and the vertical axis shows the count of the demographic / business data requested. There is also a horizontal line marking the respective average count that spans the entire graph. Finally the requested isochrone is drawn in the map area.

Both the bar chart and the results table are interactive in the sense that hovering the mouse over a specific bar or row will immediately cause the matching isochrone to be drawn on the map. One can hover the mouse in a horizontal smooth manner over the graph and observe a beautiful isochrone sequence on display, that showcases the impact of traffic (during the day's duration) to the respective isochrone coverage. Results of this impact, will be further quantified in the next section.

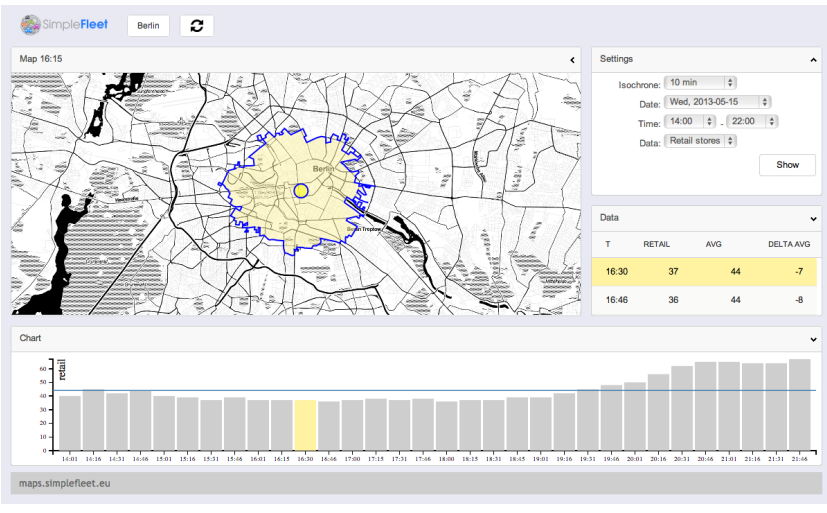

(a) Evening at 16:30

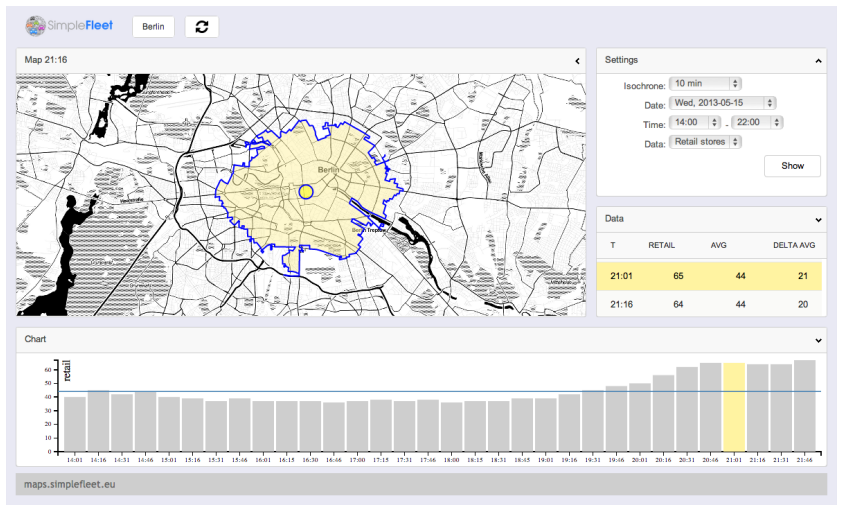

(b) Night at 21:00

Figure 2: Berlin city center, on Wed 15th May 2013. Depending on the time of the day there is a significant change in the covered area. This clearly shows the benefits of computing isochrones based on live traffic. 


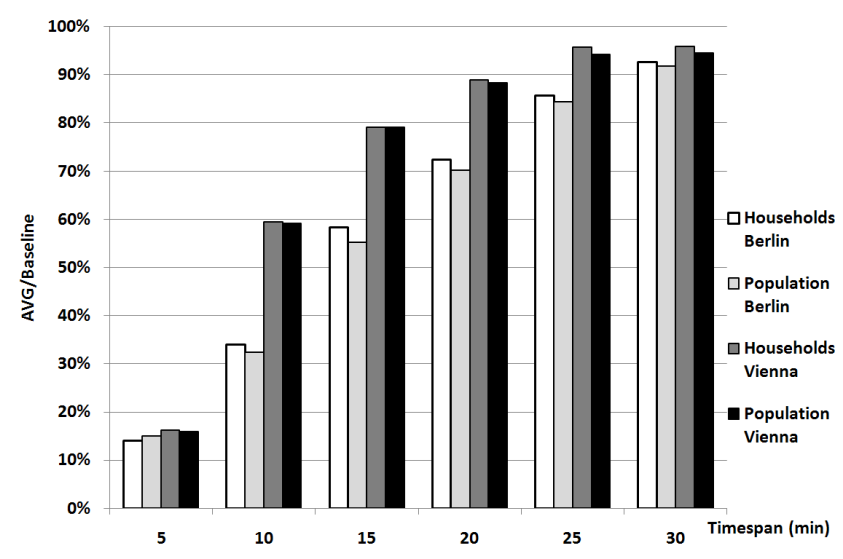

Figure 3: Average values (typical traffic situation) in comparison to traffic-less values for different timespans - isochrones

\subsection{Results}

Although our geomarketing demo clearly demonstrates the impact of traffic to typical business intelligence decisions for the urban areas of Berlin and Vienna in a concise, clear and intuitive way, it is beneficial to summarize and quantify the statistics presented in the demo. This way we may easily answer questions, such as: "To what extent does traffic affect reachability analysis" or "To what degree typical traffic-less geomarketing approaches are erroneous". The results that easily surpassed our expectations, are presented in Fig. 3 and 4.

Figure 3 shows that for both Berlin and Vienna, if we take traffic into account and for the 5 minute timespan - isochrone, we reach less than $15 \%$ of the potential customers we would have calculated on a static traffic-less road network graph. Although this gap decreases for larger timespans, still for a timespan of 15 minutes the impact of traffic is more then $40 \%$ for Berlin and $20 \%$ for Vienna, i.e., the number of actual customers we reach within $15 \mathrm{~min}$ is actually $20-40 \%$ smaller than the number calculated by the typical traffic-less scenario. If we also consider the fact that typical geomarketing applications employ convex-hull approaches (which further overestimate the respective areas, as shown in [5]), we see that our proposed approach to business intelligence issues is significantly more accurate and realistic.

This is also evident in Fig. 4 which compares the values acquired from the demo in case of a congested road network (traffic jams) with again the same baseline of a static traffic-less road network graph. Here the impact of traffic is even more dramatic, i.e., even for a timespan of 15 minutes the traffic's impact is more than $60 \%$ for both cities, i.e., the potential customers we may reach are lowered by an additional $20-45 \%$ (compared to Fig. 3) in case of traffic congestion. Since stores' opening hours usually coincide with traffic rush-hours, the worse case scenario of Fig. 4 might provide an even more realistic picture of how traffic decreases the potential pool of customers we may reach in a geomarketing decision.

\section{CONCLUSION}

In this work we have described the SimpleFleet geomarketing demo and provided all necessary information about its basic design, implementation and core functionality. Still, despite its responsiveness, ease of use and the rest of its impressive characteristics, the demo's most amazing feature is the actual results: We have shown in a concise, accurate but most of all quantitative way, the huge impact of traffic to informed business intelligence decisions.

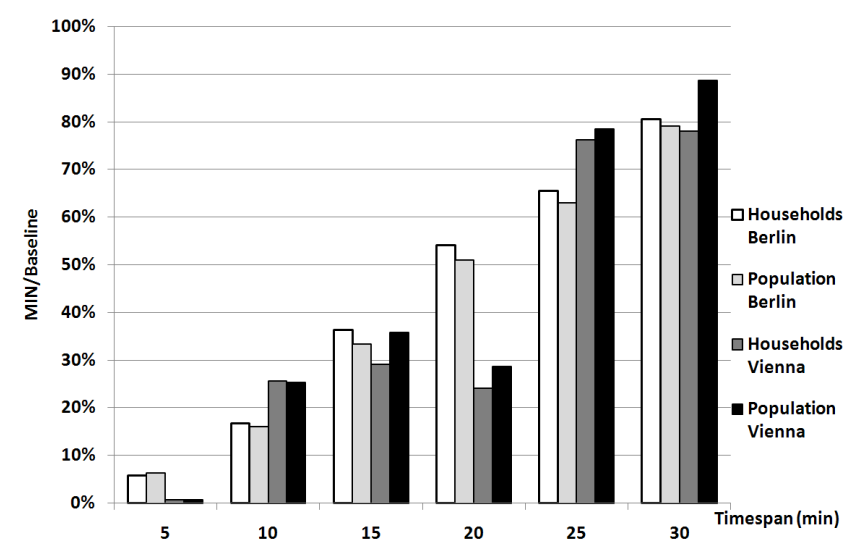

Figure 4: Minimum values (traffic-jams) in comparison to traffic-less values for different timespans - isochrones

Moreover, we have clearly described the methodology of acquiring those results, by combining live-traffic information with stateof-the-art isochrone computation and demographic / business data. At our next steps, we plan to further refine details, such as improving performance of isochrone computation or combine results with other sources of public data. But most of all, we strongly hope we may encourage other researchers to further expand our work and employ similar methodologies in different, seemingly unrelated contexts, such as crime prevention, urban planning or location-based tourist services.

\section{Acknowledgments}

The research leading to these results has received funding from the European Union Seventh Framework Programme "SimpleFleet" (http://www.simplefleet.eu, grant agreement No. FP7-ICT2011-SME-DCL-296423).

\section{REFERENCES}

[1] V. Bauer, J. Gamper, R. Loperfido, S. Profanter, S. Putzer, and I. Timko. Computing isochrones in multi-modal, schedule-based transport networks. In Proceedings of the 16th ACM SIGSPATIAL international conference on Advances in geographic information systems, GIS '08, pages 78:1-78:2, New York, NY, USA, 2008. ACM.

[2] A. Efentakis, S. Brakasoulas, N. Grivas, G. Lamprianidis, D. Pfoser, and K. Patroumpas. Towards a flexible and scalable fleet management service. Submitted.

[3] A. Efentakis and G. Lamprianidis. SimpleFleet Deliverable D6.5. SimpleFleet Online Demo [Online]. http: //www. simplefleet.eu/?page_id=84, 2013.

[4] J. Gamper, M. Böhlen, and M. Innerebner. Scalable computation of isochrones with network expiration. In Proceedings of the 24th international conference on Scientific and Statistical Database Management, SSDBM'12, pages 526-543, Berlin, Heidelberg, 2012. Springer-Verlag.

[5] S. Marciuska and J. Gamper. Determining objects within isochrones in spatial network databases. In Proceedings of the 14th east European conference on Advances in databases and information systems, ADBIS'10, pages 392-405, Berlin, Heidelberg, 2010. Springer-Verlag.

[6] SimpleFleet. Democratizing fleet management [online]. http://www.simplefleet.eu, 2013. 